\title{
Los estudiantes de ciencias, ¿pueden reconocer los argumentos lógicos involucrados en una demostración?
}

\section{Can science's students recognize the logical arguments involved in a demonstration?}

\author{
Vladímir Camacho Moreno \\ Colegio de Ciencias y Humanidades (Naucalpan)-UNAM. \\ bionica_75@yahoo.com \\ José Javier Sánchez Pozos \\ Universidad Autónoma Metropolitana - Iztapalapa \\ sanpo@xanum.uam.mx \\ Gonzalo Zubieta Badillo \\ Cinvestav (Zacatenco)-IPN \\ gzubieta@cinvestav.mx
}

RESUMEN • En toda demostración matemática, se utilizan argumentos lógicos correctos; saber reconocerlos y aplicarlos es una condición necesaria para comprender algunos de los pasos clave en las demostraciones. Pero ¿puede un estudiante identificar claramente los argumentos que han sido utilizados en una demostración? Y si los reconoce ¿puede decir cómo se utilizaron? El objetivo de este trabajo es explorar si estudiantes de Matemáticas y Computación que cuentan con experiencia en cursos universitarios, de maestría e incluso de doctorado, son capaces de identificar los argumentos usados en una demostración.

PALABRAS CLAVE: demostración; argumento lógico; prueba; transición; nivel superior.

ABSTRACT • In every mathematical proof logically correct arguments are used; it is a necessary condition to find out them, and also to recognize how they are applied, for understanding some of the key steps in the demonstrations. But can a student clearly identify the arguments used in a demonstration? And if he recognizes them, is he able to explain how they were used? The aim of this paper is to explore if mathematics' and computer science's students -who have met demonstrations at their university, master or even doctoral courses- can successfully identify and explain the arguments within a demonstration.

KEYWORDS: demonstration; logical argument; proof; transition; university level. 


\section{INTRODUCCIÓN}

Cuando los alumnos inician sus estudios en el nivel superior y han elegido una carrera en la que los contenidos matemáticos son esenciales para el buen desarrollo de esta, por ejemplo: alguna Ingeniería, Ciencias de la computación, y por supuesto, Matemáticas o Física, existen muchas dificultades que deberán enfrentar. Una de ellas es la poca o nula familiaridad que tienen con las demostraciones o pruebas matemáticas, pues se les pedirá de un momento a otro la escritura, lectura y comprensión de estas. Así, los estudiantes serán sometidos a un cambio súbito en el que la lectura de pruebas con rigor y formalidad será crucial, pues tendrán que leer sus libros de texto. Es cierto que en esta transición existen alumnos que desarrollan habilidades para leer, comprender y reproducir demostraciones, e incluso algunos de ellos llegan a crear las suyas. Desafortunadamente la gran mayoría no logra consolidar las destrezas necesarias en el transcurso de su carrera por sí mismos, es decir, sin ningún entrenamiento específico, consciente y dirigido. Por ejemplo, Jones (2000) reporta que si bien la demostración ocupa un lugar central dentro de las clases de Matemáticas en la universidad, muchos estudiantes pueden concluir sus estudios teniendo una percepción incompleta de qué constituye una prueba y cómo pueden estas desarrollarse. Este problema no solo es de aquellos estudiantes cuyo rendimiento está por debajo del promedio. Un ejemplo de ello es William Paul Thurston, ganador de la medalla Fields en 1982: "Cuando empecé como estudiante graduado en Berkeley, yo tenía problemas para imaginar cómo podría 'demostrar' un nuevo e interesante teorema matemático. Yo no entendía realmente lo que era una prueba» (Thurston, 1994).

Por otra parte, organizaciones como la NCTM (2000) en su publicación sobre los Principios y estándares para la educación matemática menciona que hoy en día al diseñar un currículo en Matemáticas debe contemplarse lo siguiente: «El razonamiento y la demostración deberían ser una parte consistente de la experiencia matemática durante toda la escolaridad». Esto ha generado diferentes perspectivas y líneas de trabajo en torno a la demostración, aumentando el interés por esta en todos los niveles. Por ejemplo, Stylianides y Stylianides (2008: 104) explican que al consultar la literatura es posible identificar fundamentalmente tres razones por las cuales el interés por la demostración se ha incrementado:

Primero, la prueba es fundamental para hacer matemáticas -es la base en la comprensión de las matemáticas y es esencial para desarrollar, establecer y comunicar el conocimiento matemático (...). Segundo, la habilidad de los estudiantes en la prueba puede mejorar su habilidad matemática de manera más amplia, porque la prueba está involucrada en todas las situaciones en donde las conclusiones han de ser alcanzadas y las decisiones tomadas (...). Tercero, (...) varios investigadores han identificado la introducción abrupta de los estudiantes a la prueba (...) como una posible explicación para las muchas dificultades que estudiantes de secundaria y universidades enfrentan con la prueba...

Moore (1994: 250), por ejemplo, menciona que aunque existen estudios empíricos relacionados con la demostración la mayoría se han desarrollado con la geometría en el nivel preuniversitario, y relativamente pocos en el nivel superior. Agrega además que la literatura sugiere las siguientes áreas como posibles fuentes de dificultades que un estudiante encuentra en el aprendizaje de la demostración: «a) percepción de la naturaleza de la prueba, $b$ ) lógica y métodos de prueba, $c$ ) habilidad para la resolución de problemas, d) lenguaje matemático, e) entendimiento del concepto». Finaliza Moore diciendo que las dificultades anteriores nos sugieren que la habilidad para leer matemáticas abstractas y hacer pruebas depende de una constelación de creencias, conocimientos y habilidades cognitivas. Sin embargo, lo anterior no es del todo claro, ni cuáles de estos factores son los más relevantes o cómo interactúan entre sí.

Dentro de las investigaciones en torno a la prueba Weber (2008: 431-432) menciona que en varios trabajos se ha señalado como un área en la cual se ha puesto poca atención, comparativamente con otras (aspectos epistemológicos y psicológicos de la demostración, sus diferentes concepciones, técnicas para su enseñanza, etc.), a la lectura de pruebas matemáticas. 
Existen estudios empíricos que muestran los beneficios que la lectura de pruebas da a los estudiantes para escribir con mayor éxito demostraciones. Por ejemplo, Powers (2010) desarrolla una investigación en un curso de Álgebra Abstracta, que se lleva a cabo con dos secciones de estudiantes. En una de estas se usan, además del contenido tradicional de Álgebra Abstracta, actividades de lectura relacionadas con la identificación entre demostraciones válidas y otras que no lo son. El otro grupo es instruido sin las actividades de lectura y es utilizado como un grupo de control. Al final del curso, los resultados revelan que los estudiantes de la sección en la que se utilizaron las actividades de lectura obtienen un mejor desempeño al escribir demostraciones.

\section{MARCO DE REFERENCIA}

\section{La lectura de pruebas}

Con el paso de los años, las investigaciones sobre la lectura de pruebas en el nivel superior se han incrementado, además de que los objetivos y las direcciones de trabajo han evolucionando, haciéndose cada vez más específicas y delimitando con mayor precisión el objeto de estudio. Por ejemplo, Selden y Selden (2003) realiza una investigación con ocho estudiantes universitarios (cuatro de matemáticas y cuatro con formación para profesor de secundaria). El objetivo del estudio es determinar si los estudiantes son capaces de identificar cuándo un argumento constituye una demostración válida. Los resultados indican que sus habilidades para realizar la tarea anterior son muy pobres. Aunque después de una entrevista reflexiva con los participantes produjo el $81 \%$ de respuestas correctas. Una investigación similar es la de Weber (2010).

Análogamente, en un estudio exploratorio de Alcock y Weber (2005), les presentaron a trece estudiantes de Matemáticas una supuesta demostración para que determinaran si era válida o no. Al final de esta supuesta demostración se hacia una afirmación verdadera, que no se seguía de manera correcta de los enunciados anteriores (aunque estos eran verdaderos también). Sin embargo, solo seis de los trece estudiantes rechazaron la prueba y únicamente dos pudieron dar razones matemáticas correctas.

También hay investigaciones con matemáticos profesionales, por ejemplo Weber $(2011,2008)$ investiga qué estrategias utilizan los matemáticos para leer demostraciones e identifica tres estrategias empleadas por ellos: apelando a la autoridad de otros matemáticos que leen la demostración; a la lectura línea por línea y a una lectura modular, es decir, por bloques. Un estudio similar se encuentra en Hanna, Jahnke y Pulte (2010: 101-111).

Las investigaciones relacionadas con la lectura de pruebas, como mencioné al principio, han evolucionado y se han hecho cada vez más puntuales, por ejemplo Mejía-Ramos e Inglis (2011) investigan la influencia que tiene el uso de ciertas palabras utilizadas cotidianamente en el lenguaje natural cuando son utilizadas en el contexto de las demostraciones matemáticas, específicamente cuando se intenta validar una demostración. Incluso se han comenzado a proponer modelos para evaluar la comprensión de una demostración en el nivel superior (Mejía-Ramos, 2012), con la finalidad de precisar mecanismos y herramientas para evaluar esta comprensión cuando un estudiante lee cierta demostración, entre otras cosas.

Existen muchos aspectos por investigar en la lectura de pruebas y, claro está, se puede hacer desde diferentes perspectivas, lo que enriquece el objeto de estudio en cuestión (la demostración). Además, esta diversidad permite delimitar y clarificar con más precisión qué elementos están en juego al leer una demostración, cómo interactúan estos y cuáles son los más relevantes, entre otras cosas. Identificar estos aspectos es indispensable para conformar metodologías y actividades de enseñanza que coadyuven eficientemente a la lectura de pruebas en el nivel universitario. 


\section{Terminología}

Cuando se habla de la demostración en matemáticas no existe una terminología aceptada por todos, es decir, algunas palabras o expresiones se utilizan como sinónimos, por ejemplo: demostrar, mostrar y probar; demostración correcta o válida; argumento correcto o válido, etc. En ocasiones, la distinción entre estos términos se hace explicando según el momento y el contexto de su uso a qué se están refiriendo. En particular, al estudiar la demostración desde el punto de vista de la Matemática Educativa se han propuesto diferentes concepciones y usos de los términos utilizados. Por ejemplo, Balacheff (2000) distingue entre explicación, prueba y demostración.

Otros investigadores como Duval (1999) y Boero (1999) hacen la distinción entre argumento y prueba, y analizan las relaciones y diferencias entre estos. Además lo hacen desde diferentes perspectivas y con supuestos diferentes. Por ejemplo, Duval distingue dos tipos de argumentaciones: las retóricas $y$ las heurísticas. Así el estudio de lo que es un argumento, sus usos y alcances tiene matices diferentes según la reconstrucción del autor que se tome.

Debido a las diferentes perspectivas y los usos de la terminología relacionada con la demostración en el ámbito de la Educación Matemática, consideramos pertinente aclarar en qué sentido usaremos en esta investigación términos como argumento, consecuencia lógica y corrección e incorrección, con la finalidad de precisar a qué nos estamos refiriendo al hacer mención de estos.

Como se mencionó en la introducción, cuando los alumnos ingresan en el nivel superior y eligen carreras como Matemáticas, Ciencias de la Computación o alguna afín a estas, desde sus primeros cursos será imprescindible que desarrollen habilidades para comprender y escribir demostraciones matemáticas. En este proceso, una de las tareas que deberán enfrentar de manera eficiente será la lectura de pruebas, específicamente aquellas presentadas en sus libros de texto. Sin embargo, las demostraciones en estos libros, en varias ocasiones, no son tan claras ya que o bien muchos pasos son omitidos, y como resultado de esto se presenta una demostración muy compacta, o bien no se explicitan algunas de las partes que son esenciales para entender y garantizar que se alcanza con claridad la comprensión de la prueba en su totalidad. Por ejemplo, en el libro de Bartle y Sherbert (1991: 43), utilizado frecuentemente en el primer semestre para la materia de Cálculo, ${ }^{1}$ se formula el siguiente teorema: ${ }^{2}$

Teorema: sean $a, b$ y $c$ elementos de $R$. Si $a \cdot b=0$, entonces $a=0$ o bien $b=0$.

Posteriormente se presenta la demostración de este, sin embargo la prueba comienza haciendo una afirmación y al final de esta se plantea la pregunta de ¿por qué?, el objetivo de la pregunta es hacer reflexionar al lector sobre el porqué de la afirmación. La demostración presentada en el libro es la siguiente.

\section{Demostración:}

Basta suponer que $a \neq 0$ y deducir que $b=0$. (¿Por qué?). Puesto que $a \cdot b=0=a \cdot 0$, se aplica el inciso $b)^{3}$ a la ecuación $a \cdot b=a \bullet 0$ para concluir que $b=0$ cuando $b=0$.

Cuando se lee la demostración anterior es necesario comprender la primera afirmación para tener claridad sobre cómo se está procediendo, es decir, por qué es suficiente suponer que $a \neq 0$ y llegar a $b=0$ para que el teorema quede probado. ¿Pero realmente los estudiantes entienden cuáles son las razones que justifican la afirmación?; en otras palabras, ¿identifican los alumnos qué argumentos

1. El nombre del curso puede variar según la institución: Análisis Matemático, Calculo Avanzado, etc.

2. En el libro, este teorema consta de tres incisos, sin embargo solo utilizaré uno para mostrar lo que deseo.

3. El $b$ ) se refiere a: si $a \bullet b=a \bullet c$ y $a \bullet 0$, entonces $b=c$. 
fueron utilizados? El tener la capacidad de clarificar los pasos, muchas veces no explícitos en una demostración, es de suma importancia para una buena comprensión, ya que como Balacheff (2000: 13) expresa: «Lo que caracteriza a las demostraciones como género del discurso es su forma estrictamente codificada. Más aún, este rigor formal debe ser matizado a la luz de la práctica. Por ejemplo, ciertas etapas de la demostración pueden no estar explícitas; el descubrirlas depende del lector». Este rigor formal no explicitado en ciertos pasos dentro de las demostraciones está relacionado en ocasiones con argumentos lógicos. Lo anterior es una característica inevitable de las matemáticas universitarias, pues en este nivel existe un mayor rigor en las clases, intrínseco de los conceptos abordados, además de una escritura rigurosa y formal. Basta con revisar un libro de los primeros semestres, digamos de Cálculo, y ver algunas definiciones (supremo, ínfimo, convergencia de una sucesión, límite de una función, etc.) para percatarse de lo anterior.

A continuación, daremos dos definiciones que utilizaré para explicar parte de la terminología utilizada, en particular la de argumento lógico.

Enunciado: es una expresión lingüística con respecto a la cual se puede decir que es verdadera o falsa, y no ambas cosas a la vez.

Argumento: es una sucesión finita de enunciados formada por premisas y la conclusión.

Sobre esta última definición ahondaremos un poco más. Tanto en la vida cotidiana como en la ciencia en general, en particular dentro de la matemática, utilizamos argumentos constantemente y los expresamos dentro de algún contexto, con una simbología particular y usando conceptos específicos del área en la que se esté trabajando, por ejemplo, parafraseando a Rosen (2004: 69):

Si $n$ es un número real tal que $n>1$, entonces $n^{2}>1$.

Supongamos que $n^{2}>1$. Concluimos entonces que $n>1$.

«Si quisiéramos clarificar el argumento liberándolo de ambigüedades y quedarnos solo con aquellos momentos que plasmen claramente el esquema del argumento (premisas y conclusión) necesitaríamos un simbolismo adecuado para esto, y ese simbolismo es el lenguaje lógico. Es decir, representar el razonamiento (...) mediante el simbolismo lógico supone 'poner en limpio' a posteriori la forma implícita del razonamiento (...). Al obrar así hemos realizado una especie de radiografía del razonamiento, un retrato de su osamenta» (Deaño, 1990: 131). Esto nos permite evidenciar la estructura lógica del argumento, lo que nos brinda la posibilidad de aplicar los métodos que la lógica ha desarrollado a través de siglos para emitir un veredicto sobre la corrección o incorrección ${ }^{4}$ de un argumento.

Por ejemplo la formalización del argumento anterior es:

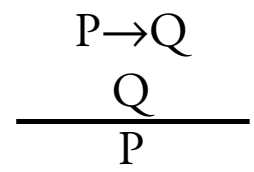

en la que P y Q (llamadas letras enunciativas) representan los enunciados P: $n$ es un número real tal que $n>1 ; \mathrm{Q}: n^{2}>1$.

Sin embargo, este argumento es incorrecto, pero si se modificara un poco ahora sería correcto:

si $n$ es un número real tal que $n>1$, entonces $n^{2}>1$.

4. Más adelante explicaré qué se entenderá por argumento correcto e incorrecto. 
Suponiendo que $n^{2} \ngtr 1$. Podemos concluir que $n \ngtr 1$. $^{5}$

Y su formalización sería:

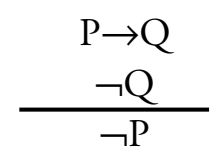

A este argumento suele llamársele modus tollendo tollens o simplemente modus tollens.

Antes de continuar, precisemos más el significado de argumento correcto e incorrecto.

La estructura general de un argumento es:

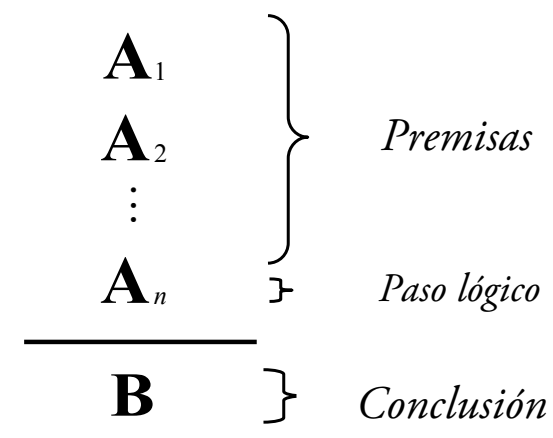

Las premisas son los enunciados que se toman como punto de partida, la conclusión es el enunciado que se obtiene como consecuencia de las premisas. La raya o línea que separa a las premisas de la conclusión es el paso lógico; este simboliza el proceso o los pasos que nos permiten pasar de las premisas a la conclusión. En palabras de Alfredo Deaño (1990: 133), «... el paso de las premisas a la conclusión la imprime -solo podría imprimirla- una regla de inferencia, un dispositivo lógico que, a partir de unas premisas con una forma determinada, arrastra una determinada conclusión». En otras palabras, son las razones que justifican cómo a partir de la forma lógica de las premisas y el suponerlas verdaderas, de manera necesaria e inexorable, se obtiene la verdad de la forma lógica de la conclusión.

El paso lógico según la complejidad del argumento puede extenderse a varios pasos. Por estar fuera del alcance y los objetivos de este trabajo, no desarrollaré todo el aparato lógico para explorar en detalle cualquier argumento. Existen muchos libros de lógica que el lector interesado puede consultar; dos excelentes obras son (Deaño, 1990) y (Copi y Cohen, 2007). A continuación, daremos la definición de consecuencia lógica y posteriormente las de argumento correcto e incorrecto.

\section{CONSECUENCIA LÓGICA}

Se dice que un enunciado $\mathbf{B}$ es consecuencia lógica de los enunciados $\mathbf{A}_{1}, \mathbf{A}_{2}, \ldots, \mathbf{A}_{n}$ si y solo si siempre que las formas lógicas de $\mathbf{A}_{1}, \mathbf{A}_{2}, \ldots, \mathbf{A}_{n}$ son verdaderas, necesariamente la forma lógica de $\mathbf{B}$ también lo es; i. e. cuando no se puede dar el caso en el que las formas lógicas de $\mathbf{A}_{1}, \mathbf{A}_{2}, \ldots, \mathbf{A}_{n}$ sean verdaderas y la de $\mathbf{B}$ sea falsa.

5. El signo $\ngtr$ representa la negación de $>$ (mayor que), es decir, $\ngtr$ es igual a $\leq$ (menor o igual que). 
Argumento correcto: un argumento es correcto si y solo si su conclusión es consecuencia lógica de sus premisas; es decir, cuando la suposición de la verdad de la forma lógica de sus premisas $\mathbf{A}_{1}, \mathbf{A}_{2}, \ldots, \mathbf{A}_{n}$ implica necesariamente la verdad de la forma lógica de su conclusión $\mathbf{B}$.

Argumento incorrecto: un argumento es incorrecto si y solo si no es correcto (si y solo si su conclusión no es consecuencia lógica de sus premisas); es decir, cuando si se puede dar el caso en que la forma lógica de sus premisas $\mathbf{A}_{1}, \mathbf{A}_{2}, \ldots, \mathbf{A}_{n}$ sea verdadera y la forma lógica de su conclusión $\mathbf{B}$ sea falsa.

De las definiciones anteriores podemos concluir que el argumento modus tollens es correcto, pero el otro:

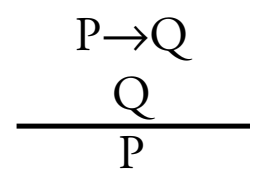

Es incorrecto, pues existe al menos un caso de valores de verdad para las letras enunciativas que hacen verdaderas las premisas y falsa la conclusión. Por ejemplo, si se toma a P como falsa y a Q como verdadera entonces ambas premisas $\mathrm{P} \rightarrow \mathrm{Q}$ y $\mathrm{Q}$ serán verdaderas, pero la conclusión $\mathrm{P}$ será falsa. Por lo tanto, el argumento es incorrecto.

Algo importante que se debe señalar es lo siguiente: con todo argumento va asociado un condicional: $\mathrm{A}_{1} \& \mathrm{~A}_{2} \& \ldots \& \mathrm{~A}_{n} \Rightarrow \mathrm{B}$ y viceversa, en el que las premisas y la conclusión forman el antecedente y el consecuente respectivamente. Muchas veces no se da el argumento explícito, sino que se expresa en forma de ley, es decir, como un enunciado, y en el caso de que el argumento representado sea correcto entonces el condicional asociado será siempre formalmente verdadero (es decir, sean cuales sean los valores de verdad de sus letras enunciativas el condicional siempre será verdadero, es decir, una tautología).

Por ejemplo, el argumento:

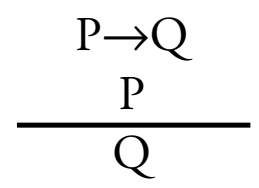

(El cual en ocasiones se toma como una regla de inferencia y se le conoce como modus ponendo ponens o simplemente modus ponens) lo podemos expresar en forma de ley: $((\mathrm{P} \rightarrow \mathrm{Q}) \& \mathrm{P}) \rightarrow \mathrm{Q}$.

\section{Aplicación de los argumentos a un ejemplo concreto}

Retomemos el enunciado del teorema y su demostración (Bartle y Sherbert, 1991: 43).

Teorema: sean $a, b$ y $c$ elementos de $R$. Si $a \cdot b=0$, entonces $a=0$ o bien $b=0$.

Demostración.

Basta suponer que $a \neq 0$ y deducir que $b=0$. (¿Por qué?). Puesto que $a \cdot b=0=a \bullet 0$, se aplica el inciso $b)^{6}$ a la ecuación $a \bullet b=a \bullet 0$ para concluir que $b=0$ cuando $a \neq 0$.

6. El $b$ ) se refiere a: si $a \bullet b=a \bullet c$ y $a \neq 0$, entonces $b=c$. 
La cuestión es si los estudiantes que leen esta demostración comprenden la primera afirmación, es decir, por qué es correcto aseverar que el teorema queda probado al suponer que $a \neq 0$ y llegar a $b=0$. En otras palabras ¿identifican qué argumentos (obviamente correctos) justifican la afirmación?

Los argumentos utilizados en la afirmación antes mencionada son:

$$
\frac{\mathrm{A} \rightarrow \mathrm{B} \vee \mathrm{C}}{\mathrm{A} \rightarrow(\neg \mathrm{B} \rightarrow \mathrm{C})} \quad \frac{\mathrm{A} \rightarrow(\neg \mathrm{B} \rightarrow \mathrm{C})}{\mathrm{A} \& \neg \mathrm{B} \rightarrow \mathrm{C}}
$$

Para hacer más explícito lo anterior, formalicemos (al nivel de la lógica de enunciados) el enunciado: si $a \cdot b=0$, entonces $a=0$ o bien $b=0$. Designando A: $a \cdot b=0 ; \mathrm{B}: a=0 ; \mathrm{y} \mathrm{C:} b=0$ su formalización es $\mathrm{A} \rightarrow \mathrm{B} \vee \mathrm{C}$. Si utilizamos el primer argumento, obtenemos como conclusión $\mathrm{A} \rightarrow(\neg \mathrm{B} \rightarrow \mathrm{C})$; es decir, si $a \cdot b=0$ entonces ( si $a \neq 0$ entonces $b=0$ ). Posteriormente, si apelamos al segundo argumento concluimos que $\mathrm{A} \& \neg \mathrm{B} \rightarrow \mathrm{C}$; es decir, si $a \bullet b=0$ y $a \neq 0$ entonces $b=0$. Finalmente se utiliza el método directo $^{7}$ para demostrar este enunciado, y las hipótesis son $a \bullet b=0$ y $a \neq 0$ y la conclusión $b=0$.

Una pregunta que puede plantearse es la siguiente: cuando los estudiantes han cursado el segundo año de su carrera o más ¿̇son capaces de reconocer los argumentos involucrados en una demostración? Concretamente me refiero a argumentos correctos como por ejemplo:
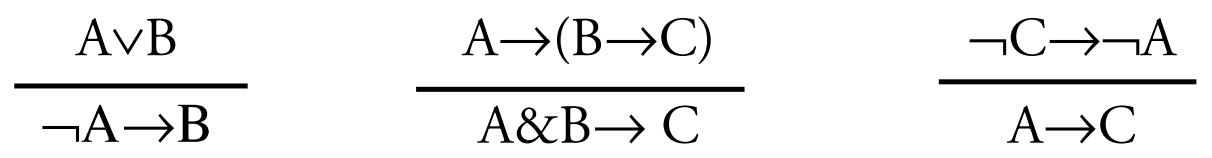

\section{Conceptualización del significado de prueba}

En esta sección, describiremos la propuesta de Andreas J. Stylianides sobre la conceptualización del significado de prueba expuesta en su artículo «Proof and Proving in School Mathematics» (Stylianides, 2007). Su definición ha sido pensada para esclarecer las partes constituyentes de una demostración como un producto final, es decir, como aquello que un individuo plasma como resultado final utilizando papel y lápiz.

Definición: prueba es un argumento matemático, una sucesión de aseveraciones para o sobre una afirmación matemática con las siguientes características:

1. usa enunciados aceptados por la comunidad del salón de clases (conjunto de enunciados aceptados) que son verdaderos y están disponibles sin mayor justificación.

2. Emplea formas de razonamiento (modos de argumentación) que son válidos y conocidos, o dentro del alcance conceptual de la comunidad del salón de clases.

3. Se comunica con las formas de expresión (modos de representación de un argumento) que son apropiadas y conocidas, o dentro del alcance conceptual de la comunidad del salón de clases.

Ejemplo de los tres componentes de un argumento matemático mencionado en la definición de prueba:

7. Si la proposición que hay que demostrar es un condicional de la forma $\mathrm{A} \rightarrow \mathrm{B}$, el método directo consiste en suponer verdadero el antecedente (A) y a partir de este llegar a establecer la verdad del consecuente (B). 
Los estudiantes de ciencias, ¿pueden reconocer los argumentos lógicos involucrados en una demostración?

\begin{tabular}{|l|l|}
\hline \multicolumn{1}{|c|}{ Componentes de un argumento } & \multicolumn{1}{|c|}{ Ejemplo } \\
\hline Conjunto de enunciados aceptados & Definiciones, axiomas, teoremas, etc. \\
\hline Modos de argumentación & $\begin{array}{l}\text { Aplicación de reglas lógicas de inferencia (tales como modus ponens y mo- } \\
\text { dus tollens), uso de definiciones para conducir a enunciados generales, enu- } \\
\text { meración sistemática de todos los casos a los que un enunciado es reducido } \\
\text { (dado que su número es finito), construcción de contraejemplos, desarrollo } \\
\text { de un razonamiento que muestra que la aceptación de un enunciado lleva a } \\
\text { una contradicción, etc. }\end{array}$ \\
\hline Modos de representación de un argumento & $\begin{array}{l}\text { Lingüísticos (por ejemplo, lenguaje oral), física, diagramática-gráfica, tabu- } \\
\text { lar, simbólico-algebraica. }\end{array}$ \\
\hline
\end{tabular}

\section{Uso de la definición}

En la definición anterior, se marcan tres aspectos que caracterizarán una prueba, identifiquemos cada uno de estos en un ejemplo concreto. Supongamos que se nos pide demostrar: si $n^{2}$ es impar entonces $n$ es impar; un estudiante contesta así:

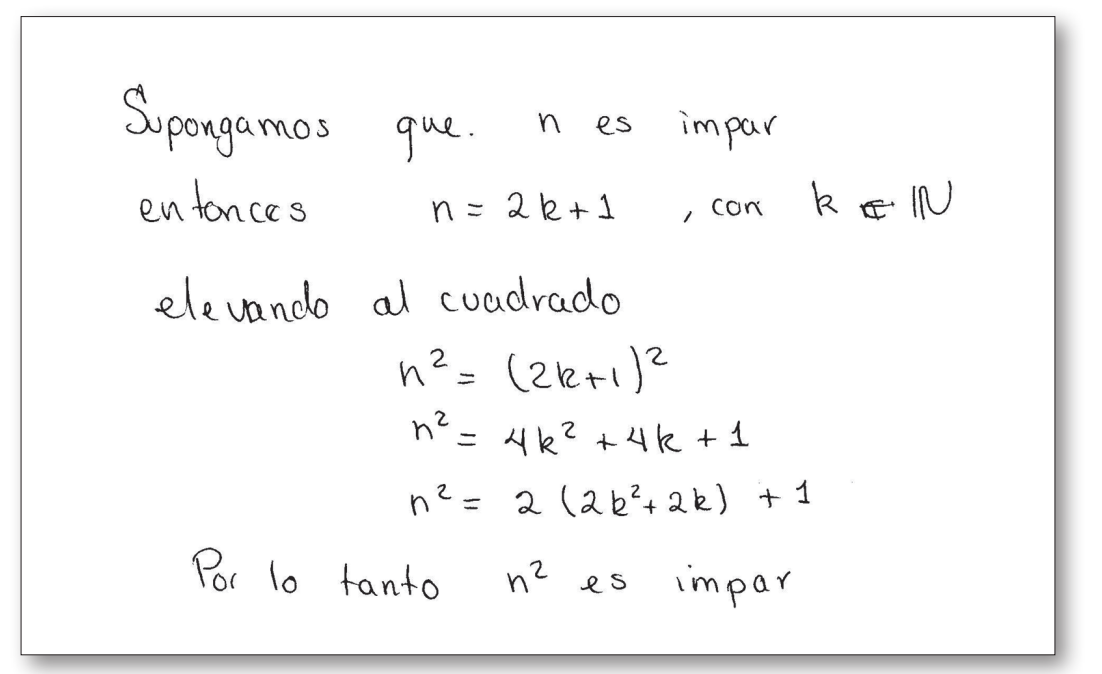

Fig. 1

Identifiquemos en esta demostración los tres puntos de la definición. Primero veamos el aspecto de conjunto de enunciados aceptados. Son aquellos supuestos válidos que se pueden utilizar, por ejemplo: un número impar se representa como $2 k+1$ con $k$ en los enteros, $(a+b)^{2}=a^{2}+2 a b+b^{2}$, etc. Respecto a este primer punto y la demostración, se podría decir, si se es muy estricto que $k$ debería pertenecer a los enteros (pues podría ser negativo), pero bueno podemos considerar que no es tan grave la falla.

Antes de analizar el segundo punto, analicemos el tercero: modos de representación de un argumento. En la presentación de la demostración, esta tiene un aspecto lingüistico, específicamente utiliza el lenguaje natural combinado con un lenguaje simbólico-algebraico, lo cual es aceptable dentro del gremio matemático.

Retomando el segundo punto, que será para nosotros el de interés (modos de argumentación), podemos decir que utiliza un argumento incorrecto en la demostración, ya que un condicional y su recíproco no son equivalentes; es decir, de $\mathbf{A} \rightarrow \mathbf{B}$ no se sigue $\mathbf{B} \rightarrow \mathbf{A}$ y viceversa. En la demostración, se utiliza el recíproco del condicional, y el participante piensa que esto prueba el condicional de partida. 
Para demostrar la incorrección de un argumento, basta con encontrar un caso de valores de verdad para las letras enunciativas (en este caso A y B) en el que las premisas sean verdaderas y la conclusión falsa. Por ejemplo, si A: verdadero y B: falsa, $\mathrm{B} \rightarrow \mathrm{A}$ será verdadera, pero $\mathrm{A} \rightarrow \mathrm{B}$ falsa; por lo tanto, el argumento anterior:

$$
\frac{\mathrm{B} \rightarrow \mathrm{A}}{\mathrm{A} \rightarrow \mathrm{B}}
$$

es incorrecto.

En la caracterización de Stylianides, se puede observar en el segundo punto (modos de argumentación) que uno de los elementos importantes es el uso de argumentos lógicos correctos, tales como: modus ponens, modus tollens, etc., pues al hacer una demostración en ciertos momentos se utilizan estos, siendo parte sustancial de la demostración.

\section{OBJETIVO Y RELEVANCIA}

«En los últimos 30 años, se han incrementado significativamente las investigaciones en el ámbito de la justificación y las demostraciones. Aunque estas investigaciones se han centrado en una amplia gama de temas, incluyendo la construcción de la prueba, la naturaleza epistemológica de la prueba, y el desarrollo de la prueba en las aulas de matemáticas, solo recientemente la investigación en educación matemática investigó la lectura de la prueba matemática» (Weber, 2011: 329). Debido a esto y a lo expuesto anteriormente, nos planteamos el siguiente objetivo: analizar las justificaciones que utilizan algunos estudiantes de Matemáticas y Computación al leer demostraciones, especificamente en aquellos pasos en los que se involucra un argumento correcto.

Existen todavía muchos interrogantes por descubrir y contestar. Además nuestro trabajo no está centrado en analizar un proceso global, como se ha realizado en otros estudios, sino en investigar un aspecto particular, intrínseco y poco abordado en los estudios sobre la demostración, la identificación de argumentos lógicos correctos.

Para diseñar actividades que mejoren la enseñanza de la demostración, es de vital importancia poder identificar y delimitar con suficiente claridad qué dificultades enfrentan los estudiantes cuando emiten juicios sobre los argumentos lógicos involucrados en ella. Así los profesores tendrán más herramientas para generar actividades de instrucción que favorezcan su comprensión. Además ellos podrían percibir la importancia de escribir sus notas de clase, textos, etc., poniendo énfasis en aquellos pasos clave para comprender una demostración, en particular en la que se involucra un argumento correcto. Es decir, evidenciar lo que Selden y Selden (1995: 129) llaman el marco de la prueba, que no depende de los conocimientos detallados de los conceptos matemáticos correspondientes.

\section{METODOLOGÍA}

\section{El cuestionario y el alcance de la investigación}

Para la recolección de datos, utilizaremos un diseño transversal. «Los datos se recogen, sobre uno o más grupos de sujetos, en un solo momento temporal; se trata del estudio en un determinado corte puntual en el tiempo, en el que se obtienen las medidas a tratar», (Buendía Eisman, 1998: 134). Emplearemos como instrumento el cuestionario; en este se presentan tres demostraciones escritas, es decir, ya hechas 
y cinco preguntas de respuesta abierta que están relacionadas con ciertos aspectos de las pruebas. Una característica importante del cuestionario es que las demostraciones no fueron elegidas ad hoc, sino que son de los libros de texto que los estudiantes utilizan en sus cursos. Además para la elección de las demostraciones se tomó en cuenta que las tres involucraran argumentos lógicos correctos. Esto se hizo con la finalidad de reforzar e incrementar la certidumbre de nuestros resultados sobre su desempeño al enfrentar argumentos lógicos.

La primera de las tres demostraciones en el cuestionario fue seleccionada del libro de Bartle y Sherbert (1991: 43), e incluía la pregunta que está en el primer renglón (¿por qué?), que hace alusión al por qué de la afirmación; es decir, la pregunta no es nuestra sino que en el libro se plantea así. Además nos pareció importante dejarla tal y como se presenta en el libro para que los estudiantes enfrenten una situación real de sus libros de texto. La segunda demostración también es uno de los ejercicios propuestos en el mismo libro de Bartle y Sherbert (p. 53), y las preguntas correspondientes están planteadas al final de la prueba. Finalmente la tercera y última demostración es del libro de Rosen (2004: 63) y la pregunta correspondiente también se presenta al final.

La aplicación del cuestionario con los estudiantes se llevó a cabo en un salón de clases, y antes de que lo contestaran se les comentó que la intención de este no era que detectaran errores conceptuales o algún paso incorrecto; esta observación se hizo con la finalidad de evitar que los participantes pensaran que el objetivo era encontrar posibles fallas en las demostraciones. Además se les indicó claramente que el objetivo era determinar y explicitar los argumentos lógicos involucrados en las pruebas. En caso de que tuvieran dudas sobre las preguntas o los teoremas presentados, se les aclararían personalmente. El tiempo que se les dio a los participantes para contestar fue de una hora, aunque nadie utilizó ese tiempo en su totalidad, pues la mayoría terminaba en un tiempo de entre 20 y 35 minutos.

A continuación, presentamos el cuestionario aplicado. Aunque en un principio este consta de tres demostraciones, solo presentaremos los resultados y el análisis de la primera, ya que esta ilustra el desempeño general de los estudiantes respecto a los argumentos lógicos involucrados, pues sus respuestas fueron parecidas en las otras dos, es decir, de naturaleza similar.

\section{DEMOSTRACIONES VÁLIDAS}

Nombre Carrera

Las siguientes demostraciones son válidas, sin embargo en cada una de ellas no se menciona la justificación de uno o varios pasos. Responde las preguntas correspondientes en cada caso.

1. Si $a \cdot b=0$ entonces $a=0$ o bien $b=0$.

\section{Demostración}

Basta suponer que $a \neq 0$ y deducir que $b=0$. (¿Por qué?). ${ }^{8}$ Puesto que $a \cdot b=0=a \bullet 0$, se aplica el inciso $b)^{9}$ a la ecuación $a \bullet b=a \bullet 0$ para concluir que $b=0$ cuando $a \neq 0$.

2. Si $a, b \in R$ probar que si $a^{2}+b^{2}=0$ entonces $a=0$ y $b=0$.

\section{Demostración}

Caso 1:

si $a \neq 0, a^{2} \neq 0$. Para cualquier $b$ se cumple que $b^{2} \geq 0$. Por lo tanto, $a^{2}+b^{2} \neq 0$.

8. En esta parte del cuestionario, se le dejaba al alumno un espacio para que él contestara el porqué del cuestionamiento.

9. El $b$ ) se refiere a: si $a \bullet b=a \bullet c$ y $a \neq 0$, entonces $b=c$. 
Caso 2:

este caso es análogo al anterior, pero con $b \neq 0$.

¿Por qué se obtienen dos casos?

¿Por qué en cada caso se prueba que $a^{2}+b^{2} \neq 0$ ?

3. Demostrar que los siguientes enunciados son equivalentes:

a) $n$ es un entero par;

b) $n-1$ es un entero impar;

c) n2es un entero par.

\section{Demostración}

a) $\Rightarrow$ b)

Supongamos que $n$ es par, entonces $n=2 k$ para algún entero $k$.

Así, $n-1=2 k-1=2(k-1)+1$ donde $k-1$ es un entero. Por lo tanto, $n-1$ es impar.

b) $\Rightarrow$ c)

Supongamos que $n-1$ es impar, entonces $n-1=2 k+1$ para algún entero $k$, es decir $n=2 k+2$. Así, $n^{2}=(2 k+2)^{2}=2\left(2 k^{2}+4 k+2\right)$ donde $\left(2 k^{2}+4 k+2\right)$ es un entero. Por lo tanto, $n^{2}$ es par.

c) $\Rightarrow \mathrm{a})$

Supongamos que $n$ es impar, entonces $n^{2}=(2 k+2)^{2}=2\left(k^{2}+2 k\right)+1$ donde $k^{2}+2 k$ es un entero. Por lo tanto, $n^{2}$ es impar.

¿Por qué al probar los condicionales a) $\Rightarrow b), b) \Rightarrow c)$ y c) $\Rightarrow a$ ) se obtiene la equivalencia de los tres enunciados?

Nuestra investigación es de tipo exploratoria, cuya característica es «... indagar sobre temas y áreas desde nuevas perspectivas» (Hernández Sampieri, 2006: 101), además también es descriptiva, pues seleccionamos «... una serie de cuestiones y se mide o recolecta información sobre cada una de ellas, para así (valga la redundancia) describir lo que se investiga» (Hernández Sampieri, 2006: 102).

\section{Participantes}

En nuestra investigación participan quince estudiantes: ocho de Matemáticas, siete de Computación. De los ocho estudiantes de Matemáticas, uno está cursando el doctorado, tres están en maestría y cuatro en el tercer año de la licenciatura. De los estudiantes de Computación, cuatro están en la maestría $\mathrm{y}$ tres en el tercer año de la licenciatura.

\section{Respuestas al cuestionario}

A continuación, presentaremos los resultados de los argumentos involucrados en las demostraciones del cuestionario correspondientes a las preguntas planteadas.

Argumentos en la prueba 1: en la página 9, se explica cuáles y cómo se utilizaron los argumentos. 
Argumentos en la prueba 2:

al argumento $\frac{\mathrm{A} \rightarrow(\mathrm{B} \& \mathrm{C})}{\neg(\mathrm{B} \& \mathrm{C}) \rightarrow \neg \mathrm{A}}$ le corresponde el condicional: $\mathrm{A} \rightarrow(\mathrm{B} \& \mathrm{C}) \Rightarrow \neg(\mathrm{B} \& \mathrm{C}) \rightarrow \neg \mathrm{A}$

(contraposición del condicional). ${ }^{10}$

Análogamente:

a $\quad \frac{\neg(\mathrm{B} \& \mathrm{C})}{\neg \mathrm{B} \vee \neg \mathrm{C}}$ le corresponde $\neg(\mathrm{B} \& \mathrm{C}) \Rightarrow \neg \mathrm{B} \vee \neg \mathrm{C}$ (negación de la conjunción);

b $\frac{(\neg \mathrm{B} \vee \neg \mathrm{C}) \rightarrow \neg \mathrm{A}}{(\neg \mathrm{B} \rightarrow \neg \mathrm{A}) \&(\neg \mathrm{C} \rightarrow \neg \mathrm{A})}$ le corresponde $(\neg \mathrm{B} \vee \neg \mathrm{C}) \rightarrow \neg \mathrm{A} \Rightarrow(\neg \mathrm{B} \rightarrow \neg \mathrm{A}) \&(\neg \mathrm{C} \rightarrow \neg \mathrm{A})$ (por casos).

En la prueba 2, la respuesta a la pregunta: ¿por qué se obtienen dos casos? se debe a la aplicación de los tres argumentos anteriores, lo cual describiremos brevemente. Formalicemos el condicional: si $a^{2}+b^{2}=0$, entonces $a=0$ y $b=0$; simbolizando $a^{2}+b^{2}=0$ con $\mathrm{A} ; a=0$ con B: y $b=0$ con C, obtenemos $\mathrm{A} \rightarrow(\mathrm{B} \& \mathrm{C})$. Utilizando el primer argumento, concluimos que $\neg(\mathrm{B} \& \mathrm{C}) \rightarrow \neg \mathrm{A}$. Posteriormente en el antecedente de este condicional (el contrapuesto) aplicamos la negación de la conjunción, obteniendo $(\neg \mathrm{B} \vee \neg \mathrm{C}) \rightarrow \neg \mathrm{A}$, es decir, si $a \neq 0$ o $b \neq 0$, entonces $a^{2}+b^{2} \neq 0$. Finalmente, si aplicamos el argumento por casos a $(\neg \mathrm{B} \vee \neg \mathrm{C}) \rightarrow \neg \mathrm{A}$ concluimos lo siguiente $(\neg \mathrm{B} \rightarrow \neg \mathrm{A}) \&(\neg \mathrm{C} \rightarrow \neg \mathrm{A})$, es decir, (si $a \neq 0$ entonces $\left.a^{2}+b^{2} \neq 0\right)$ y $\left(\right.$ si $b \neq 0$ entonces $\left.a^{2}+b^{2} \neq 0\right)$. Hemos obtenido dos condicionales diferentes unidos por una conjunción que tienen que demostrarse. ${ }^{11}$ En la segunda pregunta, ¿por qué en cada caso se prueba que $a^{2}+b^{2} \neq 0$ ?, se debe al uso del método directo para demostrar los condicionales correspondientes, por ejemplo: en el condicional si $a \neq 0$ entonces $a^{2}+b^{2} \neq 0$, se toma como hipótesis $a \neq 0$ y se concluye que $a^{2}+b^{2} \neq 0$. Análogamente al otro.

Argumentos en la prueba 3:

¿por qué la prueba de los condicionales a) $\Rightarrow$ b), b) $\Rightarrow c$ ) y c) $\Rightarrow$ a) conlleva a la equivalencia de los tres enunciados a), b) y c)?

Debido al argumento: $\frac{(\mathrm{A} \rightarrow \mathrm{B}) \&(\mathrm{~B} \rightarrow \mathrm{C}) \&(\mathrm{C} \rightarrow \mathrm{A})}{(\mathrm{A} \leftrightarrow \mathrm{B}) \&(\mathrm{~B} \leftrightarrow \mathrm{C}) \&(\mathrm{C} \leftrightarrow \mathrm{A})}$,

tiene lugar $(\mathrm{A} \rightarrow \mathrm{B}) \&(\mathrm{~B} \rightarrow \mathrm{C}) \&(\mathrm{C} \rightarrow \mathrm{A}) \Rightarrow(\mathrm{A} \leftrightarrow \mathrm{B}) \&(\mathrm{~B} \leftrightarrow \mathrm{C}) \&(\mathrm{C} \leftrightarrow \mathrm{A})$, donde $\mathrm{A}, \mathrm{B}$ y $\mathrm{C}$ representan: $n$ es un entero par; $n$-1es un entero impar y $n^{2}$ es un entero par, respectivamente.

Otra respuesta sería: $(\mathrm{A} \rightarrow \mathrm{B}) \&(\mathrm{~B} \rightarrow \mathrm{C}) \&(\mathrm{C} \rightarrow \mathrm{A}) \Leftrightarrow(\mathrm{A} \leftrightarrow \mathrm{B}) \&(\mathrm{~B} \leftrightarrow \mathrm{C}) \&(\mathrm{C} \leftrightarrow \mathrm{A})$.

Haremos uso de la siguiente nomenclatura para identificar a los estudiantes: $M$, estudiante de Matemáticas; C, estudiante de Computación. Los subíndices en las letras corresponderán al grado que cursan: $d$, doctorado; $m$, maestría; $t$, tercer año de la carrera. Así, ' $\mathrm{M}_{d}$ ' representa un estudiante de Matemáticas en doctorado; ' $\mathrm{C}_{t}$ ', estudiante de Computación del tercer año; etc. Además ' $\mathrm{M}_{m}=3$ ' denotará tres estudiantes de Matemáticas en maestría; $\mathrm{M}_{d}=1$, un estudiante de Matemáticas en doctorado, etcétera. Por lo tanto, tenemos: $\mathrm{M}_{d}=1 ; \mathrm{M}_{m}=3 ; \mathrm{M}_{t}=4 ; \mathrm{C}_{m}=4 ; \mathrm{C}_{t}=3$.

10. Estas representaciones son consecuencia de que a todo argumento se le puede asociar un condicional vid. supra, p. 8 .

11. Una conjunción es verdadera si y solo si sus dos conyuntos son verdaderos. 


\section{RESULTADOS}

Las respuestas que los participantes dieron a la pregunta de ¿por qué? se pueden ubicar básicamente en cuatro tipos de respuestas. Es importante mencionar que esta ubicación podría traslaparse en algún momento, es decir, no son necesariamente disjuntas dos a dos. En otras palabras, no es una clasificación o categorización en el sentido estricto del término. Sin embargo, la intención de esta ubicación es delimitar algunos rasgos distintivos de naturaleza parecida.

Tipo 1. Las respuestas ubicadas aquí utilizan el hecho de que el consecuente ( $a=0$ o bien $b=0)$ es una disyunción, argumentando que si esta es verdadera entonces uno de los disyuntos será verdadero ( $b=0$, lo cual es cierto), pero afirmando que el otro es falso $(a \neq 0)$, lo cual no necesariamente es cierto, pues se puede dar el caso de que los dos disyuntos sean verdaderos ( $a=0$ y $b=0){ }^{12}$

Respuesta $\mathrm{C}_{m}$ :

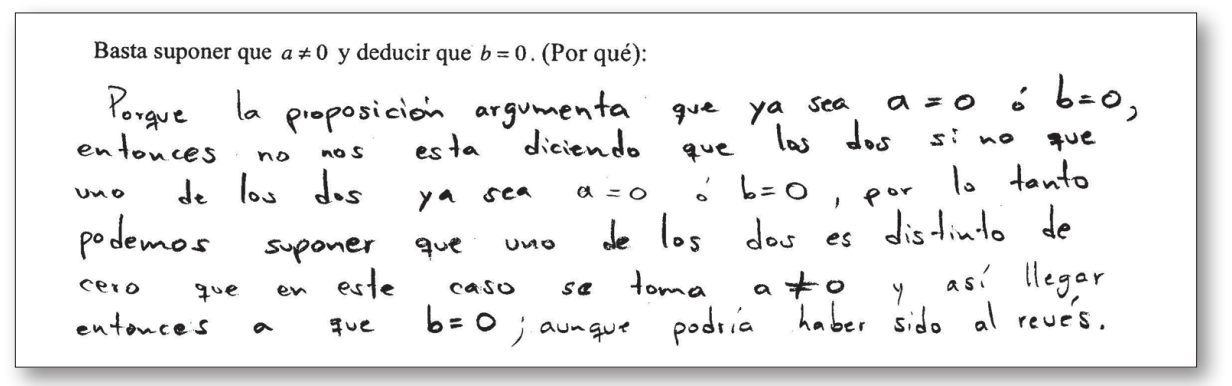

Fig.2

Respuesta $\mathrm{M}_{m}$ :

Basta suponer que $a \neq 0$ y deducir que $b=0$. (Por qué):

El otro coso seria sopener $b \neq 0$ \& $a=0$ pero la demostincion

Seria la misma havendo un intercambio entre $a_{y} b$.

Fig. 3

Tipo 2. Las respuestas situadas aquí son aquellas cuya falla radica en utilizar el hecho que se desea establecer (petitio principii), además de la siguiente propiedad de los números reales: a $9=0$ para cualquier $a$, o bien ambas.

Respuesta $\mathrm{C}_{m}$ :

$$
\begin{aligned}
& \text { Basta suponer que } a \neq 0 \text { y deducir que } b=0 \text {. (Por qué): } \\
& \text { Hay un producto de dos cantidades, con que una de ellas valga } O \text {, es } \\
& \text { condrción suficiente para que el resultado de la multiplicación sea } 0 \text {, } \\
& \text { y no importa tue valor tunga } a \text {. }
\end{aligned}
$$

Fig. 4

12. Una disyunción es verdadera si y solo si al menos uno de los disyuntos es verdadero. 
Respuesta $\mathrm{C}_{i}$ :

Basta suponer que $a \neq 0$ y deducir que $b=0$. (Por qué):

Sabemos que cualquier numero multiplicado por

cero da como resultado cero, de tal manera que

no importa que valor tenga ' $a$ ', si $b=0$ se

cumple la condición mencionzda.

Fig. 5

Respuesta $\mathrm{C}_{i}$ :

Basta suponer que $a \neq 0$ y deducir que $b=0$. (Por qué):

Parque se necesita que $a=0$ ó $b=0$ en este

caso $b=0$ para aue se puedademostrar

que $a \cdot b=0$

Fig. 6

Tipo 3. En este tipo estará la respuesta de un solo participante, específicamente $\mathrm{M}_{d}$. Él se apoya para su respuesta en el antecedente y el consecuente del condicional. Sin embargo, más que dar una justificación de la afirmación inicial en la demostración, él ofrece razones de por qué al suponer que $a \neq 0$ s e puede concluir que $b=0$

Respuesta $M_{D}$ :

Basta suponer que $a \neq 0$ y deducir que $b=0$. (Por qué):

Coma el cansearente es una disyunaion o $a=0$ a bren $b=0$.

Suponiondo que $a \neq 0$ ent. $a \cdot b=0(\operatorname{con} a \neq 0)$

Por ha prop. de dominio antero se orgue que $b=0$.

Fig. 7 
Tipo 4. Aquí ubicaremos también, como en el Tipo 3, solo la respuesta de un participante, él fue el único que utilizó argumentos lógicos.

Respuesta $M_{t}$ :

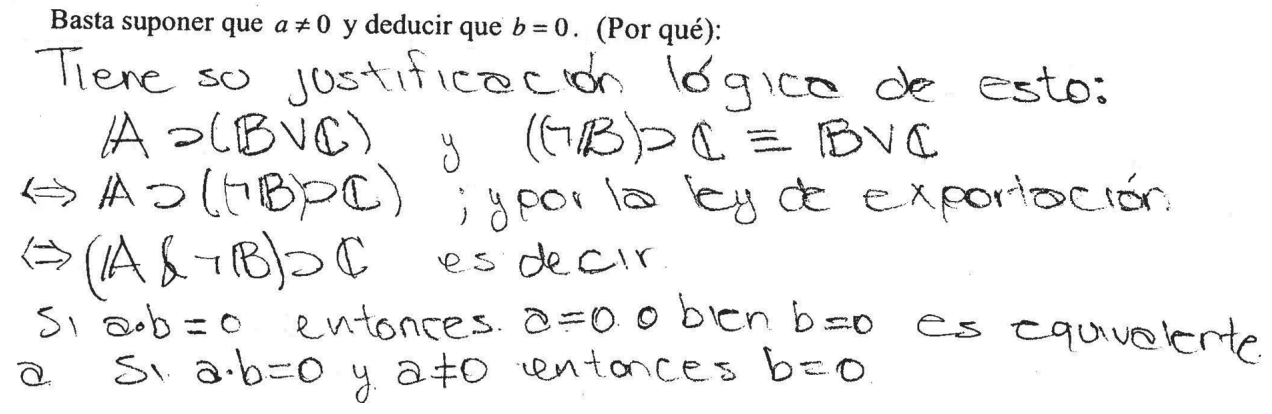

Fig. 8

Explicaremos con más detalle cuál fue el proceder de este participante. Primero mencionaremos que tradicionalmente, en los cursos de Matemáticas, para simbolizar la implicación material se utiliza: ' $\rightarrow$ o ' $\Rightarrow$ ' o bien ' $\supset$ ' (lo cual también es correcto); además utiliza ' $\equiv$ ' para denotar la equivalencia. Él simboliza (implícitamente) de la siguiente forma $\mathrm{A}: a \bullet b=0 ; \mathrm{B}: a=0 \mathrm{y} C: b=0$, y obtiene $\mathrm{A} \supset(\mathrm{B} \vee \mathrm{C})$. Además señala que $(\neg B \supset C)$ es equivalente a $(B \vee C)$, es decir, $(\neg B \supset C) \equiv(B \vee C)$. Aplicando esta equivalencia al consecuente del condicional $\mathrm{A} \supset(\mathrm{B} \vee \mathrm{C})$, se obtiene $\mathrm{A} \supset(\neg \mathrm{B} \supset \mathrm{C})$; posteriormente utiliza la ley de importación (aunque en la respuesta del estudiante el nombre es incorrecto), cuya simbolización es $(\mathrm{A} \supset(\neg \mathrm{B} \supset \mathrm{C})) \equiv((\mathrm{A} \& \neg \mathrm{B}) \supset \mathrm{C})$, concluyendo $(\mathrm{A} \& \neg \mathrm{B}) \supset \mathrm{C}$. Finalmente, el estudiante menciona que el enunciado si $a \bullet b=0$ entonces $a=0$ o bien $b=0$, es equivalente a si $a \bullet b=0$ y $a \neq 0$ entonces $b=0$.

$\mathrm{Al}$ preguntarle por qué hasta ahí deja su respuesta, él contesta: «Es claro que después de llegar a esta equivalencia se procede utilizando el método directo para hacer la demostración, suponiendo primero que $a \neq 0$ ».

\begin{tabular}{|l|c|c|c|c|c|}
\hline & $\mathrm{M}_{d}$ & $\mathrm{M}_{m}$ & $\mathrm{M}_{\mathrm{t}}$ & $\mathrm{C}_{m}$ & $\mathrm{C}_{t}$ \\
\hline Tipo 1 & & 3 & 1 & 3 & 1 \\
\hline Tipo 2 & & & 2 & 1 & 2 \\
\hline Tipo 3 & 1 & & & & \\
\hline Tipo 4 & & & 1 & & \\
\hline
\end{tabular}

\section{ANÁLISIS DE RESULTADOS Y CONCLUSIONES}

Comenzaremos diciendo que la mayoría de las respuestas están ubicadas dentro de los Tipos 1 y 2 con ocho y cinco respuestas, respectivamente. De los quince participantes, trece contestaron acorde a las características de los Tipos antes mencionados. Respecto a los Tipos 3 y 4, estos solo tienen una respuesta asignada.

Como primer punto, podemos decir que las respuestas de Tipo 1 enfatizan la frase «o es una o es otra», haciendo referencia a que $a \neq 0$ y $b=0$ o bien $a=0$ y $b \neq 0$.

Los participantes interpretan que no se puede dar el caso de que $a$ y $b$ sean iguales a cero. Un rasgo distintivo de las respuestas de este tipo es la de inferir que de la verdad de una disyunción forzosamente 
uno de los disyuntos es falso, lo cual es totalmente incorrecto. Sin embargo, esta inferencia es el eje conductor de su justificación a la pregunta de ¿por que?

En las respuestas de Tipo 2, se utiliza el hecho que se desea establecer (petitio principii) o que $a \bullet 0=0$ para cualquier $a$, o bien ambos. Los participantes piensan que el enunciado «basta suponer que $a \neq 0$ y deducir que $b=0$ » es uno de los tres posibles casos en los que el producto $a \bullet b=0$ se cumple: $(a=0$ y $b=0),(a \neq 0$ y $b=0)$ o bien $(a=0$ y $b \neq 0)$, y que basta demostrar uno de los tres para que el teorema quede probado. Aunque el caso en el que los dos son cero ni lo analiza, posiblemente porque lo consideran obvio. Llama la atención que incluso dos estudiantes de matemáticas del tercer año cometen este error.

En el Tipo 3, está ubicada la respuesta del estudiante de Matemáticas que cursa actualmente su doctorado $\left(\mathrm{M}_{d}\right)$. Con él se tuvo una entrevista personal para que nos explicara con más detalles su respuesta y nos dijo lo siguiente: "Como lo que se quiere demostrar es una disyunción, podemos suponer que $a \neq 0$ y tenemos además por hipótesis que $a \bullet b=0$, por lo tanto de esto debemos obtener forzosamente que $b=0$, porque en la disyunción al menos uno tiene que ser verdadero, es decir, cero». Cuando le preguntamos «¿por qué eso que nos dices justifica la afirmación?», él se quedó pensando por un momento (dudando) y contestó: «Bueno, con el tiempo he aprendido a proceder así, principalmente de mis profesores; sé que estoy bien y siempre lo utilizo con éxito en mis demostraciones, pero no sé exactamente por qué es correcto; aunque intuyo que se debe a que en la disyunción al menos uno de los enunciados tiene que ser verdadero». Como puede observarse en su respuesta, la experiencia y el uso le ha enseńado a proceder correctamente, sin embargo él no tiene ni la claridad ni la certeza total sobre qué justifica la afirmación hecha en la demostración; además él menciona «siempre me ha funcionado».

Finalmente la respuesta situada como de Tipo 4 fue en la única que explícitamente se clarificaron los argumentos lógicos utilizados, y aunque se hace de manera muy compacta, al final se escribe la equivalencia del enunciado original.

En las respuestas de los participantes, puede observarse cómo se apela constantemente al contenido y a las intuiciones sobre el manejo de los conceptos. Con esto no queremos decir que usar nuestras intuiciones sea malo o erróneo, sino que son insuficientes. Además esas intuiciones, en ocasiones, no son del todo correctas; por ejemplo, en las respuestas del Tipo 1 los estudiantes utilizan sus intuiciones para interpretar la disyunción, sin embargo no lo hacen del todo bien. El ocupar solo intuiciones para interpretar ciertas expresiones matemáticas puede llevarnos a tener problemas con el razonamiento matemático formal, pues como menciona Epp (2003: 888): «Ciertas formas de las declaraciones están abiertas a diferentes interpretaciones en el conocimiento informal, y el conocimiento del mundo suele determinar cuál interpretación aceptar dentro de una gama de posibilidades».

Otra constante en la mayoría de las respuestas, como ya se ha dicho, es pretender contestar haciendo referencia solo al contenido, lo cual nuevamente es insuficiente, ya que en muchas ocasiones la justificación de las afirmaciones no depende de la verdad o la falsedad de lo que se está diciendo (es decir, del contenido), sino del cómo se está diciendo (la forma lógica). Así, la mayoría de nuestros participantes utilizaron únicamente la intuición y el conocimiento del contenido como herramientas para justificar los pasos en los que aparecen argumentos correctos.

Es importante volver a enfatizar que ni el uso de las intuiciones ni el uso del contenido es malo y mucho menos incorrecto, pero sí es insuficiente en varios casos; particularmente cuando el objetivo es identificar argumentos correctos o incorrectos y, claro está, cuando uno mismo intenta construir los propios.

El acostumbrarse o solo conocer y manejar como herramientas las intuiciones que uno posee y el contenido involucrado puede llevarnos a cometer errores. Por ejemplo, si quisiéramos demostrar el recíproco del enunciado si $a \bullet b=0$ entonces $a=0$ o bien $b=0$, es decir, si $a=0$ o $b=0$ entonces $a \bullet b=0$, sabiendo que el argumento: 


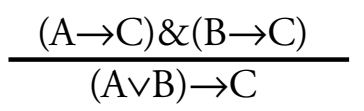

es correcto, entonces sería suficiente demostrar que $(a=0 \rightarrow a \bullet b=0) \&(b=0 \rightarrow a \bullet b=0)$ para que el condicional quedara demostrado. ${ }^{13}$ Sin embargo, si utilizáramos el argumento incorrecto:

$$
\frac{(\mathrm{A} \rightarrow \mathrm{C}) \vee(\mathrm{B} \rightarrow \mathrm{C})}{(\mathrm{A} \vee \mathrm{B}) \rightarrow \mathrm{C}}
$$

- pensando que es correcto- y lo aplicáramos para demostrar el mismo condicional, entonces afirmaríamos erróneamente: es suficiente demostrar que $(a=0 \rightarrow a \bullet b=0) \vee(b=0 \rightarrow a \bullet b=0)$ para demostrar el enunciado si $a=0$ o $b=0$ entonces $a \bullet b=0$. Esto nos podría llevar a pensar lo siguiente: como es suficiente que un disyunto sea verdadero para que la disyunción sea verdadera, entonces es suficiente demostrar que $b=0 \rightarrow a \bullet b=0$ para que $(a=0 \rightarrow a \bullet b=0) \vee(b=0 \rightarrow a \bullet b=0)$ sea verdadera, y de esto último inferir el condicional $a=0 \vee b=0 \rightarrow a \bullet b=0$. Claro que sí basta demostrar que $b=0 \rightarrow a \bullet b=0$ para establecer la verdad de la disyunción $(a=0 \rightarrow a \bullet b=0) \vee(b=0 \rightarrow a \bullet b=0)$, pero es incorrecto decir que de esto se sigue la verdad del condicional $a=0 \vee b=0 \rightarrow a \bullet b=0$, pues el argumento utilizado es incorrecto. Es necesario probar tanto $(\mathrm{A} \rightarrow \mathrm{C})$ como $(\mathrm{B} \rightarrow \mathrm{C})$ para poder concluir que $(\mathrm{A} \vee \mathrm{B}) \rightarrow \mathrm{C}$.

Una cuestión importante sobre los resultados del cuestionario es que los participantes no eran estudiantes novatos, sino con cierta experiencia universitaria, incluso algunos de ellos con experiencia en posgrado (maestría o doctorado). Además las preguntas eran sobre contenidos ya estudiados en su primer año de la carrera. Esto nos podría hacer pensar que un alumno puede ir aprobando los cursos y no comprender cabalmente muchas de las demostraciones, al menos aquellas en las que se involucran argumentos lógicos, denominadas de rutina, y que aparecen en sus libros de texto.

Las razones de esto pueden ser muchas. Una posible es que cuando los estudiantes están en sus cursos los profesores no expliciten los argumentos involucrados en las pruebas, ya sea por tiempo, porque lo obvian, o incluso podría suceder que ellos tampoco los pudieran explicitar. Alvarado Monroy y González Astudillo (2010: 74) mencionan: «Entre el profesor y los estudiantes se constituyen de manera interactiva aunque no explícita ciertas normas sociomatemáticas». Y agrega a este comentario que estas normas «determinan la actividad que se realiza diariamente y, en concreto, las que regulan la argumentación matemática, de aquí surgen, por ejemplo, las creencias que tienen los alumnos acerca de cuándo una explicación matemática se puede considerar aceptable o qué se entiende por justificación». Termina diciendo: «Otro de estos acuerdos se refiere a la construcción de un aparato lógico y lingüístico suficiente para abordar los problemas matemáticos».

Lo anterior nos hace pensar que muchas veces no se es lo suficientemente explícito en el desarrollo de habilidades lógicas, en particular en la identificación de argumentos correctos, menos en la de los incorrectos, dentro de las demostraciones, y mucho menos en su aplicación concreta cuando se escribe una.

$\mathrm{Y}$ aunque con el tiempo una persona puede desarrollar habilidades para proceder de manera correcta - por ejemplo, el alumno de doctorado- no necesariamente sabrá con claridad por qué se puede proceder así. De esta manera, cuando a él le toque el turno de impartir clases tampoco sabrá explicar con exactitud por qué son correctas ciertas afirmaciones, procedimientos o algunos momentos clave en una demostración.

13. A: $a=0 ; \mathrm{B}: b=0$ y C: $a \cdot b=0$. 
Los resultados anteriores abren nuevas perspectivas e interrogantes, por ejemplo: los profesores que forman a nuestros alumnos ¿sí saben con claridad qué argumentos están involucrados en una demostración? ¿Qué justificaciones dan los alumnos y profesores si los argumentos tienen cuantificadores?, etcétera. Estas y otras cuestiones relacionadas con argumentos correctos en una demostración necesitan más investigación con la finalidad de poder delimitar con mucha mayor precisión cuáles son los obstáculos que hay que enfrentar. Posteriormente sería factible generar un conjunto de actividades que ayuden a superar estos obstáculos. Incluso se podría iniciar una seria reflexión sobre cómo escribir los textos de matemáticas en los que se incluyen demostraciones pues, como concluye en uno de sus puntos Ibañes y Ortega (2004: 29): «La ausencia de intención didáctica que se observa en el tratamiento que dan lo libros de texto a la demostración, que se concreta en la uniformidad de métodos y estilos, en el silencio sobre sus funciones, en las casi inexistentes reflexiones sobre la naturaleza del procedimiento, en la ausencia de explicaciones sobre la expresiones que se utilizan, en la unánime carencia de explicaciones sobre el sentido global del proceso y de sus líneas maestras (...) debería ser tenido en cuenta para remediarla en lo posible».

\section{REFERENCIAS BIBLIOGRÁFICAS}

Alcock, Lara y Keith Weber (2005). Proof validation in real analysis: inferring and checking warrants. Mathematics Behavior, 24(2), pp. 125-134.

http://dx.doi.org/10.1016/j.jmathb.2005.03.003

Alvarado Monroy, Angelina y M. ${ }^{a}$ Teresa González Astudillo (2010). La implicación lógica en el proceso de demostración matemática: estudio de un caso. Enseñanza de las ciencias, 28(1), pp. 73-84.

Balacheff, Nicolas (2000). Procesos de prueba en los alumnos de matemáticas. Colombia: Universidad de los Andes.

Bartle, Robert G. y Donald R. Sherbert (1991). Introducción al Análisis Matemático. México: Limusa Noriega Editores.

Boero, Paolo (1999). Argumentación y demostración: una relación compleja, productiva, e inevitable en las matemáticas y en la educación matemática. Disponible en línea: <http://www-didactique. imag.fr/preuve/Newsletter/990708Theme/990708ThemeES.html> (consulta el 10 noviembre del 2012).

Buendía Eisman, Leonor, et al. (1998). Métodos de Investigación en Psicopedagogía. México: McGrawHill.

Copi, Irving M. y Carl Cohen (2007). Introducción a la Lógica. México: Limusa Noriega Editores.

DeAÑo, Alfredo (1990). Introducción a la lógica formal. España-Madrid: Alianza Universidad Textos.

Duval, Raymond (1999). Argumentar, demostrar, explicar: ¿continuidad o ruptura cognitiva? México: Pitagora Editrice Bologna y Grupo Editorial Iberoamérica.

Epp, Susanna S. (2003). The Role of Logic in Teaching Proof. The American Mathematical Monthly, $110(10)$, pp. 479-488.

Jones, Keith (2000). The student experience of mathematical proof at university level. International Journal of Mathematical Education in Science and Technology, 31(1), pp. 53-60. http://dx.doi.org/10.1080/002073900287381

Hanna, Gila, Hans Niels Jahnke y Helmut Pulte (eds.) (2010). Explanation and Proof in Mathematics: Philosophical and Educational Perspectives. USA-New York: Springer.

http://dx.doi.org/10.1007/978-1-4419-0576-5 
Hernández Sampieri, Roberto et al. (2006). Metodología de la Investigación. México: McGraw-Hill.

IbAÑEs, M. y T. Ortega (2004). Un análisis del tratamiento de la demostración matemática en los libros de texto de Bachillerato. Números, 57, pp. 19-32.

Mejía-Ramos, Juan Pablo y Matthew Inglis (2011). Semantic contamination and mathematical proof: can a non-proof prove? Journal of Mathematical Behavior, 30(1), pp. 19-29.

http://dx.doi.org/10.1016/j.jmathb.2010.11.005

Mejía-Ramos, Juan Pablo, et al. (2012). An assessment model for proof comprehension in undergraduate mathematics. Educational Studies in Mathematics, 79(1), pp. 3-18.

http://dx.doi.org/10.1007/s10649-011-9349-7

Moore, Robert C. (1994). Making the transition to formal proof. Educational Studies in Mathematics, 27(3), pp. 249-266.

http://dx.doi.org/10.1007/BF01273731

NCTM (National Council of Teachers of Mathematics) (2000). Principios y estándares para la educación matemática. Sevilla: Thales.

Powers, Robert A. et al. (2010). Impact of proof validation on proof writing in abstract algebra. International Journal of Mathematical Education in Science and Technology, 41(4), pp. 501-514. http://dx.doi.org/10.1080/00207390903564603

Rosen, Keeneth H. (2004). Matemática discreta y sus aplicaciones. España: McGraw-Hill.

Selden, Annie y John Selden (2003). Validations of Proofs Considered as Texts: Can Undergraduates Tell Whether an Argument Proves a Theorem? Journal for Researche in Mathematics Education, 34(1), pp. 4-36.

http://dx.doi.org/10.2307/30034698

Selden, John y Annie Selden (1995). Unpacking the logic of mathematical statements. Educational Studies in Mathematics, 29(2), pp. 123-151.

http://dx.doi.org/10.1007/BF01274210

Stylianides, Gabriel J. y Andreas J. Stylianides (2008). Proof in School Mathematics: Insights from Psychological Research into Students' Ability for Deductive Reasoning. Mathematical Thinking and Learning, 10(2), pp. 103-133.

http://dx.doi.org/10.1007/BF01274210

Stylianides, Andreas J. (2007). Proof and proving in school mathematics. Journal for Researche in Mathematics Education, 38(3), pp. 289-321.

Thurston, W. P. (1994). Back to classics: teaching limits through infinitesimals. Bulletin American Mathematical Society, 27(2), pp. 161-177. http://dx.doi.org/10.1090/S0273-0979-1994-00502-6

Weber, Keith (2011). Why and how mathematicians read proof an exploratory study. Educational Studies in Mathematics, 76(3), pp. 329-344. http://dx.doi.org/10.1007/s10649-010-9292-z

Weber, Keith (2010). Mathematics Majors' Perceptions of Conviction, Validity, and Proof. Mathematical Thinking and Learning, 12(4), pp. 306-336. http://dx.doi.org/10.1080/10986065.2010.495468

Weber, Keith (2008). How mathematicians determine if an Argument is a valid proof. Journal for Researche in Mathematics Education, 39(4), pp. 431-459. 


\section{Can science's students recognize the logical arguments involved in a demonstration?}

\author{
Vladímir Camacho Moreno \\ Colegio de Ciencias y Humanidades (Naucalpan)-UNAM. \\ bionica_75@yahoo.com
}

\author{
José Javier Sánchez Pozos \\ Universidad Autónoma Metropolitana - Iztapalapa \\ sanpo@xanum.uam.mx
}

\author{
Gonzalo Zubieta Badillo \\ Cinvestav (Zacatenco)-IPN \\ gzubieta@cinvestav.mx
}

First year university students who choose a major where mathematical contents are essential for a good performance in that major, like Engineering, Computer Science, Physics and Mathematics, will have to face a number of difficulties. One of them is that they have little or no previous knowledge of mathematical demonstrations and they will be asked to write, read or solve them. Therefore, students will need to undergo a sudden change in which the rigorous and formal reading of proofs, mainly those presented in their text books, will be decisive. However, the demonstrations in those books are not clear sometimes, considering that some steps are taken out and that the demonstrations presented are very short; or maybe some parts are not explicit, which can make it difficult to understand the proof as a whole.

This lack of rigor in some of the steps of the demonstration is sometimes related to formal logical arguments, for instance, given the following argument:

If $n$ is a real number such that $n>1$, then $n^{2}>1$.

Supposing that $n^{2} \ngtr 1$. We can conclude that $n \ngtr 1 .{ }^{1}$

The formalization is:

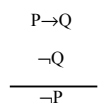

where P and Q (called statement letters) represent the formulation P: $n$ is a real number such that $n>1$; Q: $n^{2}>1$, and the formalization would be:

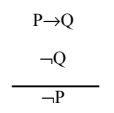

An example would be the following theorem presented in Bartle and Sherbert's book (1991, p.43), which is often used in the first semester of Calculus ${ }^{2}$ :

Theorem: Given $a, b$ and $c$ as elements of $R$. If $a \cdot b=0$, then $a=0$ or $b=0$.

They present the demonstration. However, when they present the proof, they use an affirmation and at the end they ask the question why? The objective of asking that question is to make readers reflect on the reason for the affirmation. The demonstration presented in the book is as follows:

Demonstration:

We suppose that $a \neq 0$ and we deduce that $b=0$. (Why?) Given that $\mathrm{a} \cdot \mathrm{b}=0=\mathrm{a} \cdot 0$, we apply part $b)^{3}$ to the equation $a \cdot b=a \cdot 0$ to conclude that $b=0$ when $a \neq 0$.

We can ask if students who read that demonstration understand the first affirmation, that is to say, why is it correct to affirm that the theorem is proven when we suppose that $a \neq 0$ and we come to $b=0$ ? In other words, can students identify which logical arguments (obviously correct) justify the affirmation?

The arguments used in the above affirmation are:

1. The sign $\ngtr$ means the negation of $>$ (more than), that is, $\ngtr$ is equal to $\leq$ (less or equal than).

2. The name of the course can change depending on the institution: Mathematical Analysis, Advanced Calculus, etc.

3. ' $b$ )' refers to: If $a \cdot b=a \cdot c$ and $a \neq 0$, so $b=c$. 


$$
\frac{\mathrm{A} \rightarrow \mathrm{B} \vee \mathrm{C}}{\mathrm{A} \rightarrow(\neg \mathrm{B} \rightarrow \mathrm{C})} \quad \frac{\mathrm{A} \rightarrow(\neg \mathrm{B} \rightarrow \mathrm{C})}{\mathrm{A} \& \neg \mathrm{B} \rightarrow \mathrm{C}}
$$

To be more explicit, we can formalize (on a logical level) the formulation: If $a \cdot b=0$, then then $a=0$ or $b=0$. Designing A: $a \cdot b=0$; $\mathrm{B}: a=0$; and $\mathrm{C}: b=0$ its formalization is $\mathrm{A} \rightarrow \mathrm{B} \vee \mathrm{C}$. Using the first argument we can get to the conclusion $\mathrm{A} \rightarrow(\neg \mathrm{B} \rightarrow \mathrm{C}$ ); this is, if $a \cdot b=0$ then (if $a \neq 0$ then $b=0$ ).

Then, appealing to the second argument, we can conclude that $A \& \neg B \rightarrow C$; this is,

If $a \cdot b=0$ and $a \neq 0$ then $b=0$. Finally, we use the Direct Method to show this formulation, and the hypotheses are $a \cdot b=0$ and $a \neq 0$ and the conclusion is $b=0$

The question we can ask is: when students have already taken their second year at university or further, are they able to recognize the arguments involved in a demonstration? Specifically, we refer to the correct arguments like:

$$
\frac{\mathrm{A} \vee \mathrm{B}}{\neg \mathrm{A} \rightarrow \mathrm{B}} \quad \frac{\mathrm{A} \rightarrow(\mathrm{B} \rightarrow \mathrm{C})}{\mathrm{A} \& \mathrm{~B} \rightarrow \mathrm{C}} \quad \frac{\neg \mathrm{C} \rightarrow \neg \mathrm{A}}{\mathrm{A} \rightarrow \mathrm{C}}
$$

Therefore, the main objective of this paper is: to analyze the justifications that some mathematics and computing students use when they read demonstrations, especially in those steps where a correct argument is involved.

BARTLE, ROBERT G and SHERBERT, DONALD R. (1991). Introducción al Análisis Matemático. México: Limusa Noriega Editores.

4. If the proposition to be demonstrated is a condition like $\mathrm{A} \rightarrow \mathrm{B}$, the Direct Method consists of supposing that the antecedent is true (A) and from this we can establish the truth of the consequent (B). 